\title{
Final Report Sustained Spheromak Physics Project FY 1997 - FY 1999
}

\author{
E. B. Hooper and D. N. Hill
}

February 29, 2000

U.S. Department of Energy

Lawrence

Livermore

National

Laboratory 


\section{DISCLAIMER}

This document was prepared as an account of work sponsored by an agency of the United States Government. Neither the United States Government nor the University of California nor any of their employees, makes any warranty, express or implied, or assumes any legal liability or responsibility for the accuracy, completeness, or usefulness of any information, apparatus, product, or process disclosed, or represents that its use would not infringe privately owned rights. Reference herein to any specific commercial product, process, or service by trade name, trademark, manufacturer, or otherwise, does not necessarily constitute or imply its endorsement, recommendation, or favoring by the United States Government or the University of California. The views and opinions of authors expressed herein do not necessarily state or reflect those of the United States Government or the University of California, and shall not be used for advertising or product endorsement purposes.

Work performed under the auspices of the U.S. Department of Energy by the University of California Lawrence Livermore National Laboratory under Contract W-7405-Eng-48.

This report has been reproduced directly from the best available copy.

Available to DOE and DOE contractors from the

Office of Scientific and Technical Information

P.O. Box 62, Oak Ridge, TN 37831

Prices available from (423) 576-8401 http://apollo.osti.gov/bridge/

Available to the public from the National Technical Information Service

U.S. Department of Commerce 5285 Port Royal Rd., Springfield, VA 22161

http://www.ntis.gov/

OR

Lawrence Livermore National Laboratory Technical Information Department's Digital Library

http://www.llnl.gov/tid/Library.html 


\section{Final Report \\ Sustained Spheromak Physics Project}

FY 1997 - FY 1999

Tracking \# 97-SI-009

Principal Investigators: $\quad$ E. B. Hooper and D.N. Hill

$\begin{array}{lll}\text { Funding: } & \text { FY1997 } & \$ 750 \mathrm{k} \\ & \text { FY1998 } & \$ 1050 \mathrm{k} \\ & \text { FY1999 } & \$ 1217\end{array}$

Report Outline

1. Executive Summary

2. Experimental Program Goals

3. The SSPX Experiment

3.1 Coaxial injector, flux conserver, bias coils, and power systems

3.2 Plasma diagnostics

4. Experimental results

5. Present Status and Future Directions

Appendix 1. Selected References

Appendix 2. Collaborations 


\section{Executive Summary}

This is the final report on the LDRD SI-funded Sustained Spheromak Physics Project for the years FY1997-FY1999, during which the SSPX spheromak was designed, built, and commissioned for operation at LLNL. The specific LDRD project covered in this report concerns the development, installation, and operation of specialized hardware and diagnostics for use on the SSPX facility in order to study energy confinement in a sustained spheromak plasma configuration. The USDOE Office of Fusion Energy Science funded the construction and routine operation of the SSPX facility. The main distinctive feature of the spheromak is that currents in the plasma itself produce the confining toroidal magnetic field, rather than external coils, which necessarily thread the vacuum vessel.

There main objective of the Sustained Spheromak Physics Project was to test whether sufficient energy confinement could be maintained in a spheromak plasma sustained by DC helicity injection. Achieving central electron temperatures of several hundred $\mathrm{eV}$ would indicate this. In addition, we set out to determine how the energy confinement scales with $T_{e}$ and to relate the confinement time to the level of internal magnetic turbulence. Energy confinement and its scaling are the central technical issues for the spheromak as a fusion reactor concept. Pending the outcome of energy confinement studies now under way, the spheromak could be the basis for an attractive fusion reactor because of its compact size, simply-connected magnetic geometry, and potential for steady-state current drive.

During the first year of this LDRD project (FY1997), effort focused on optimizing the spheromak design for physics performance. The optimization included MHD calculations of equilibria, evaluation of MHD stability, and determination of the dependence of these on the flux conserver dimensions and shape. (The flux conserver is the conducting wall surrounding the plasma; currents flowing in the wall provide the magnetic field for the spheromak equilibrium and determine the stability to the dominant MHD modes.) In addition, a new magnetic field geometry was developed which incorporated additional magnets to produce a vertical bias field to allow formation of a flux-core spheromak. The basic design for the capacitor bank and pulseforming network required to sustain the spheromak plasma was also developed during this period. Finally, it was determined that detailed internal magnetic field profiles 
would be required to understand the relationship between energy confinement and the plasma dynamo sustaining the current, so a collaboration with UC Davis was initiated in order to develop Ultra-short-pulse reflectometry as a means for internal field profile measurements.

In FY1998 design activities were completed and hardware procurements started on a number of key elements needed for the sustainment experiments. The design details for the coaxial helicity injector and flux conserver were nailed down and the components went out for fabrication. Very early in the year, we changed the design significantly to reduce thermal stresses in key injector components, which then led to a change in the vacuum bakeout requirements. The surface conditioning plans were subsequently modified and the bake system hardware went out for procurement. During this time we began work on an initial set of diagnostics needed for energy confinement studies, including an array of magnetic probes, Rogowski coils, and highspeed visible camera systems. In parallel with the work on the hardware, we improved the CORSICA code to better simulate spheromak formation, and we ran the code to optimize diagnostic designs for MHD reconstruction.

Energy confinement experiments using the SSPX device began in the third quarter of FY1999, following the completion of the experimental facility earlier that fiscal year. A photo of the SSPX facility as of November 1999, appears in Fig. 1. During initial experiments, we studied the breakdown process in the coaxial source. We found it very sensitive to the magnetic configuration and we developed several novel approaches to improving this first part of the spheromak discharge.

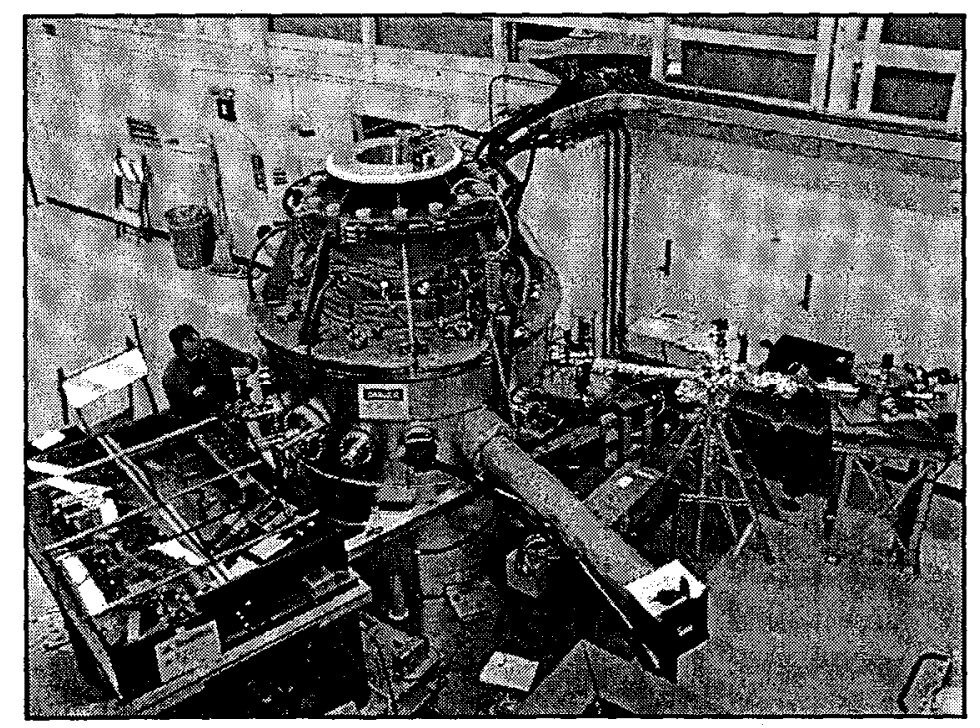

Fig. 1. Photograph of the SSPX device at LLNL (11/8/99). External components are plasma diagnostics. 
Following the breakdown experiments, we began a systematic study of spheromak formation, beginning with mapping out the formation threshold current to compare with theory. Good agreement was found, but we learned that the injector does not capture all of the flux in the gun and this must be taken into account to explain the formation threshold. As part of this study, we used the CORSICA code to reconstruct the MHD equilibrium consistent with edge magnetics data; during the decaying phase, the field structure is consistent with the Taylor-state predicted by theory. We also carried out some preliminary experiments to simulate operation with a flux-core configuration.

During FY1999 we expanded the diagnostic set available for spheromak formation experiments and worked to improve vacuum conditions with the addition of titanium gettering. We also designed and built a set of six bias magnetic field coils to produce flux-core spheromak configurations. Additional diagnostics brought on line during this time included bolometer arrays, gated TV cameras to look for asymmetries, extra Rogowski coils for measuring wall currents, and an ion-doppler visible spectrometer. A Thomson scattering system to measure density and temperature profiles was installed using OFES money. The data throughput for the data acquisition system was increased when we upgraded the computer system.

So, in summary, at the end of FY1999, we have an operating spheromak experiment which has sufficient diagnostic capability to carry out basic energy confinement studies during spheromak formation and decay. We found that the injector is capturing somewhat less flux than expected, though the energy efficiency of the system is in the expected range. Progress on sustainment experiments was less than planned due to hardware difficulties, but we did carry out some preliminary simulation experiments by splitting the formation bank into two parts. At this point, we are well positioned to carry out these experiments using OFES funding and plan to carry out experiments with the bias field coils at the end of FY2000.

\section{Experimental Program Goals}

The primary attractive feature of the spheromak concept is that currents in the plasma itself produce the confining toroidal magnetic field, rather than by external coils 
which necessarily thread the vacuum vessel. This could lead to smaller, cheaper power plants. There are three main areas of uncertainty in spheromak performance:

1. Is there sufficient energy confinement (can it hold heat)?

2 Can the configuration hold adequate plasma pressure (against pressure driven MHD modes)?

3 Can the magnetic configuration be actively controlled with external coils (against MHD tilt and shift modes)?

The central issue of energy confinement in spheromaks is tied to the fundamental mechanism which produces the force-free field configurations (Taylor states) characteristic of these devices, namely both ideal and resistive MHD modes. The transport of helicity (interconnected flux) into the core of the spheromak, and the dynamo action underlying the transport, are thought to be due to resistive "tearing" modes in the plasma. These modes break axisymmetry (as required by Cowling's theorem for the dynamo), and are closely related to magnetic reconnection events, for example in the tail of the magnetosphere and on the surface of the sun. They result when dissipative processes, typically in a thin "reconnection" layer, allow the magnetic field to find a lower energy state. When there are multiple modes undergoing this process, magnetic turbulence results. One consequence is the opening of the equilibrium magnetic surfaces, allowing energy to leak from the spheromak core to the edge.

Recent scaling studies[1], including data from previous experiments, indicate that this turbulence should weaken as the plasma becomes hot, becoming small enough at reactor temperatures that the energy losses are small. However, this scaling has not been demonstrated by experiments, and the detailed physics is not well understood. Therefore, we started the experimental spheromak program at LLNL, supported by theory, modeling, and collaborations with other experimental groups, to address this issue and, to some extent, the others outlined above. The research is carried out primarily using the SSPX (Sustained Spheromak Physics Experiment) experimental facility. Within the scope of the LDRD project, we set out to:

- Design a state-of-the-art coaxial helicity injection system for driving the spheromak. The injection system consists of both vacuum hardware and capacitor-bank power system, 
- Develop methods for conditioning the plasma-facing components that build upon the base of recent results from the tokamak community,

- Develop diagnostics and analysis techniques to measure the internal current, density, and MHD fluctuation profiles in a sustained spheromak configuration.

- Measure the energy confinement in sustained spheromak plasmas at temperatures of several hundred $\mathrm{eV}$, and determine its relationship to magnetic fluctuations.

- Test alternate magnetic geometries which should have improved drive efficiencies.

These goals form a necessary set of initial objectives which must be met in order to make progress on the spheromak concept. The LLNL spheromak program is part of an invigorated US DOE program to develop alternate paths to fusion power besides the tokamak, which is the present leading candidate for a magnetic fusion energy power reactor. The Office of Fusion Energy Science has defined a development path for concepts that begins at the "Concept Exploration " phase. At this level, experiments are funded for periods of 3-6 years at the $\$ 3-5 \mathrm{M} / \mathrm{yr}$ level in order to address a very limited set of questions fundamental to making the concept work. As stated above, global energy confinement scaling is the key issue for the spheromak.

In the remainder of this report, we summarize the results of this project in terms of meeting our objectives.

\section{The SSPX Spheromak}

The SSPX spheromak device was designed and built at LLNL. It began operating in April 1999. The device is similar to other coaxial injection spheromaks such as CTX and SPHEX, but several significant improvements have been incorporated into the design, including a conformal flux conserver shape to minimize open field lines, a flexible magnetic geometry, and a large-radius injector to increase drive efficiency. State-of-theart vacuum techniques have been used throughout to minimize impurities in the plasma. Figure 2 shows a cross section of the device and labels the main components: vacuum tank, flux conserver, inner electrode, and outer electrode. A reference MHD plasma equilibrium generated by the CORSICA code is included. 


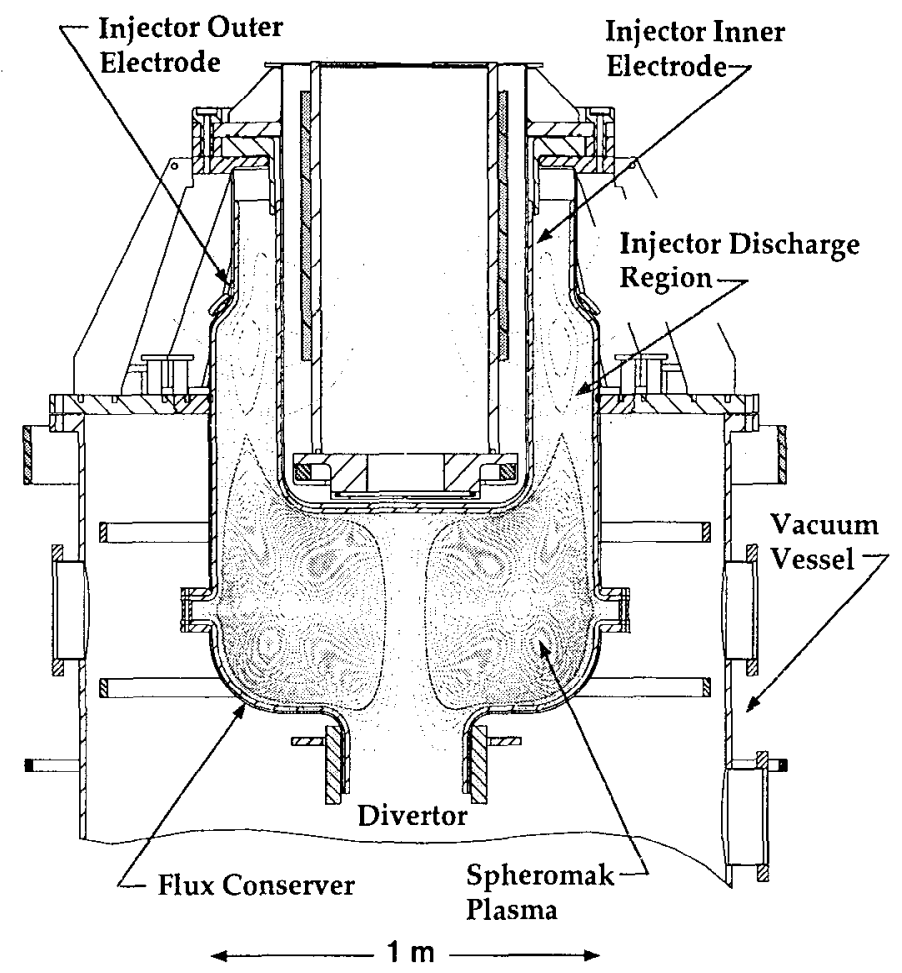

Fig. 2. Spheromak in vacuum vessel, showing the helicity injector (inner and outer electrodes) flux conserver, injector coils inside the injector inner electrode, bias field coils and representative spheromak plasma magnetic flux surfaces calculated by the CORSICA code.

The SSPX device has been designed to produce $2 \mathrm{msec}$ plasma pulses with peak toroidal currents as high as 1.5MA and peak toroidal fields in the range of 1-1.5Tesla. To date, plasma currents of 0.5MA have been obtained with pulse lengths of just over $1 \mathrm{msec}$. The total stored energy available from the capacitor banks is $2.0 \mathrm{MJ}$.

\subsection{The SSPX injector, flux conserver, and power systems}

A major component in the first half of the LDRD project was the physics analysis and design necessary to define the requirements for the two key elements of the SSPX facility, the spheromak injector and flux conserver. These studies included optimizations of the following: the flux conscrver gcometry, which must hold plasmas stable to tilt and shift modes; the plasma divertor (to handle particles and energy reaching the plasma edge), which influences the magnetic geometry; plasma current profiles, which also affect the magnetic geometry; and the magnetic bias flux system for the chosen flux conserver geometry. These results have driven the detailed mechanical design of the flux conserver.

During the design phase, the major radius of the flux conserver was increased by $20 \%(0.4$ to $0.5 \mathrm{~m})$. This change should reduce the ratio of edge current density to plasma density, and to decrease the diffusion of field lines into the walls (helicity loss). Perhaps most significantly, the increased volume-to-surface area ratio of the larger 
system should reduce the impurity radiation losses. Increasing the diameter of the flux conserver required new MHD equilibrium calculations, using the CORSICA code, to determine the optimum shape in the curved sections.

The SSPX cross section of Fig. 2 shows the expected flux surfaces for a reference plasma with a toroidal plasma current of $1 \mathrm{MA}$. The walls of the volume form a flux conserver in which wall currents support the plasma current. The thickness of the copper flux conserver ( $>1.2 \mathrm{~cm}$ ) was chosen to so that the magnetic field diffusion time through it was much longer than the pulse length $(20 \mathrm{msec} v \mathrm{~s} .2 \mathrm{msec})$. In the lower flux conserver and on the inner electrode, we increased the thickness by about a factor of two in order to obtain sufficient strength to handle repeated application of the magnetic field pressure (as high as 15 atms). An electrical discharge in the vertical (coaxial) slot injects plasma and helicity (linked magnetic fluxes) into the flux conserver; the plasma magnetic field is sustained by a magnetic dynamo that tries to conserve the total helicity.

Spheromak physics modeling and computations were largely focused on simulating the expected SSPX plasma in order to optimize the injector and to predict expected diagnostic signals as a design tool. Other studies explored MHD stability and cnergy transport in this configuration. Our main tool was the CORSICA code, developed under a previous LDRD initiative, which solves for the equilibrium current profile (actually $\lambda=j / B$ ), given boundary conditions on the edge (the flux conserver), a model pressure profile, and the injector current distribution. During the course of the project, we improved the CORSICA model to include explicitly the helicity injector (Helicity is the measure of the coupled toroidal and poloidal magnetic fluxes which drive the current in a spheromak through a magnetic dynamo). An example of the combined spheromak-injector equilibrium, calculated using the LLNL CORSICA code is shown in Fig.3a. 
(a)

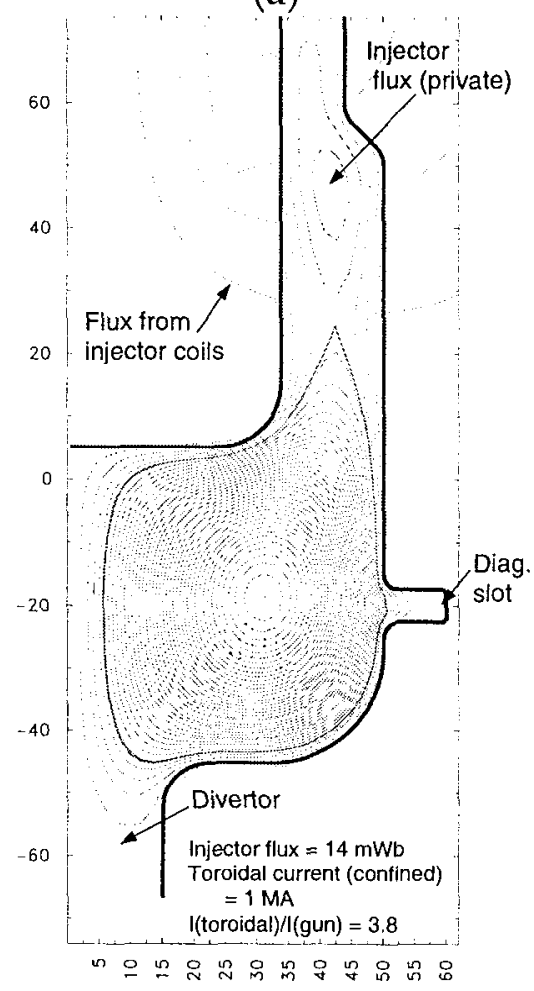

(b)

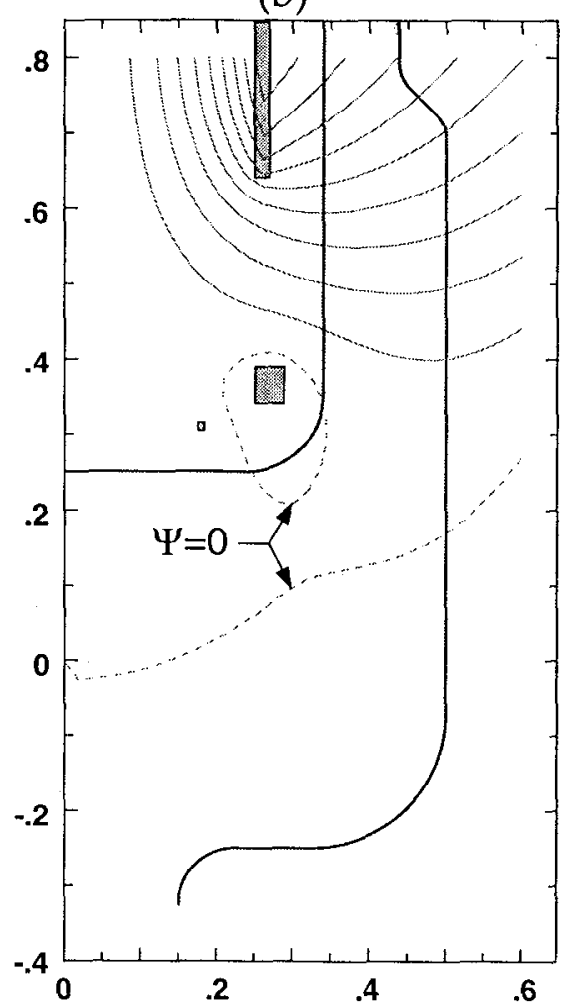

Fig. 3 (a) Magnetic equilibrium for the sustained spheromak at a toroidal current of $1 \mathrm{MA}$; dimensions in $\mathrm{cm}$. (b) Injector vacuum magnetic field; dimensions in meters. The geometry is axisymmetric about the vertical axis on the left side of the figures. In the experiment, the discharge is struck along the vacuum magnetic field lines between the inner electrode and the outer wall of the flux conserver. The resulting magnetic pressure pushes the field lines into the primary flux conserver volume and forms the toroidal configuration.

Previous equilibrium calculations have used an approximate model for the injector as one of the boundary conditions for the plasma. In the new calculations, the plasma in the injector is modeled as force-free, that is as having zero kinetic pressure and fluid flow, and it is assumed to be relaxed to a state with minimum magnetic energy at a fixed magnetic helicity. A vacuum magnetic bias field is applied (Fig. $3 \mathrm{~b}$ ). The resulting field lines are frozen into the walls of the spheromak, but in the plasma are bent by the magnetic pressure resulting from the large discharge currents. The results confirm the validity of the previous model, while eliminating approximations of the current distribution in the injector.

This equilibrium has been used to examine the effect of spheromak parameters such as the current density spatial profile and the plasma pressure. The results will provide guidance to the SSPX experiments. In addition, the equilibria have been used to model 
experimental diagnostics, especially the magnetic probes and the ultra-short pulse reflectometry discussed in Section 3.2 below.

Since impurity radiation is important to the power balance in spheromaks, we also spent considerable effort studying baking and vacuum requirements for SSPX. We considered both a high temperature bake $\left(350^{\circ} \mathrm{C}\right)$ now used on many tokamak fusion devices, and a more modest $150^{\circ} \mathrm{C}$ bakeout temperature. Bakeout temperature is usually a design driver for the vacuum system, especially in the case of spheromaks with DC helicity injection, as high voltage insulation across a vacuum seal is required. In the case of SSPX, we concluded that acceptable vacuum conditions could be obtained with the lower bakeout temperature in the neighborhood of $150-200^{\circ} \mathrm{C}$, which had the added advantage of allowing for a more robust insulator design for the helicity injector system. Typically, the system is baked for about $150 \mathrm{hrs}$, after which the water vapor is reduced below the hydrogen outgassing of the stainless steel vessel. Base pressures of $1 \times 10^{-8}$ torr with a global leak rate of $1 \times 10^{-5}$ torr- $1 /$ sec or less are obtained routinely.

Since the flux conserver and coaxial injector regions are in close contact with the plasma, we decided to coat the copper surfaces with refractory metal to minimize sputtering. Tungsten is the material of choice for such applications, but adding such a coating to a copper substrate is a challenge when the system will be baked. This is due to the difference in thermal expansion between the two materials. We investigated both electroplating (in house) and plasma spray (external vendors) procedures. We decided on plasma sprayed tungsten because of its superior adhesion properties during thermal cycling, and because it was faster and cheaper. The particular process chosen was to spray the elements in an argon environment, which was cheaper than spraying them under vacuum. The drawback to this method is that it produces a relatively porous surface which can retain oxygen; we kept the coating thin $(100 \mu \mathrm{m})$ to minimize the potential impurity inventory.

In addition to baking the system and minimizing the use of o-ring seals, we also utilize titanium gettering to reduce the impurity content. A set of 3 titanium getters has been installed in the vacuum vessel and these can be run before each plasma discharge, though we typically only use them once every $3-5$ shots. We are now in the process of evaluating the cffect of gettering on the main plasma parameters. 
Two capacitor banks power SSPX. These banks were designed to perform two very different functions. The formation bank is a high voltage, high current bank (10kV, 450kA) whose main function is to initiate the discharge, push the plasma out of the injector, and form a spheromak inside the flux conserver. The total energy in the formation bank is $0.5 \mathrm{MJ}$ and the pulse length is about $0.5 \mathrm{msec}$, depending on the plasma load impedance. A simplified schematic of the SSPX injector power system is shown in Fig. 4.

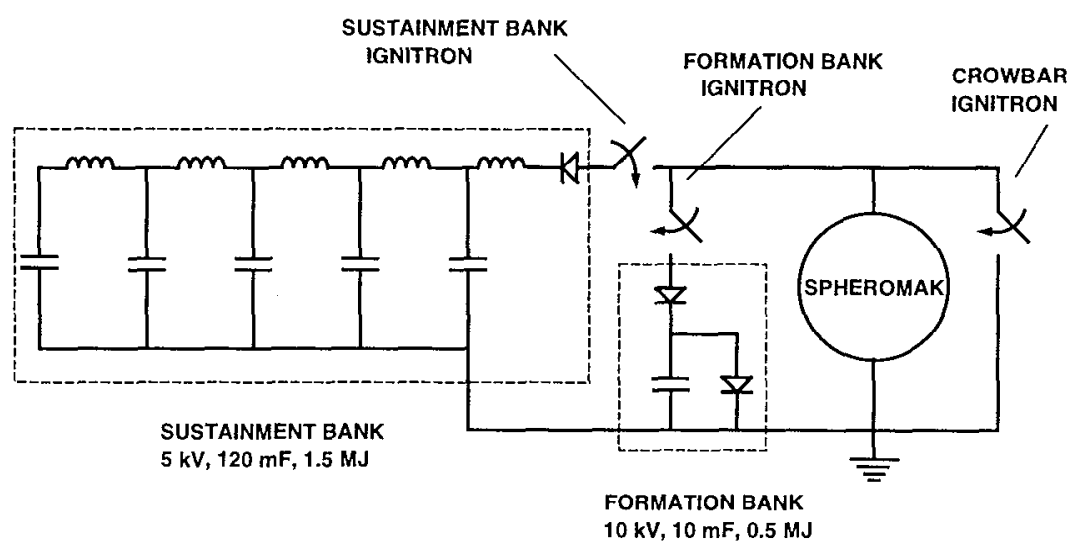

Fig. 4 Power systems schematic (simplified).

To sustain the spheromak discharge after formation, a bank of capacitors and inductor coils configured as a pulse-forming network (PFN) has been constructed to deliver a $2 \mathrm{~ms}$ pulse duration into a load of $10 \mathrm{~m} \Omega$. The sustainment bank energy is 1.5 $\mathrm{MJ}$ at $5 \mathrm{kV}$. Firing times for each bank are independently controlled by the trigger times of ignitron switch tubes. To study the decay phase of a spheromak plasma discharge, a bank of six parallel-connected ignitron switch tubes is available to shunt the capacitor bank current. Construction of this power system was completed on November 11, 1998. Subsequent testing showed that the circuit performed as designed; though the plasma load impedance was lower than expected. Thus, several of the system parameters (ballast resistance and formation-bank source inductance) were modified to improve the match and increase the energy delivered to the load. During final testing, several connections to the sustainment bank failed and a redesign is presently underway. Fig. 5 shows the completed SSPX capacitor banks. 


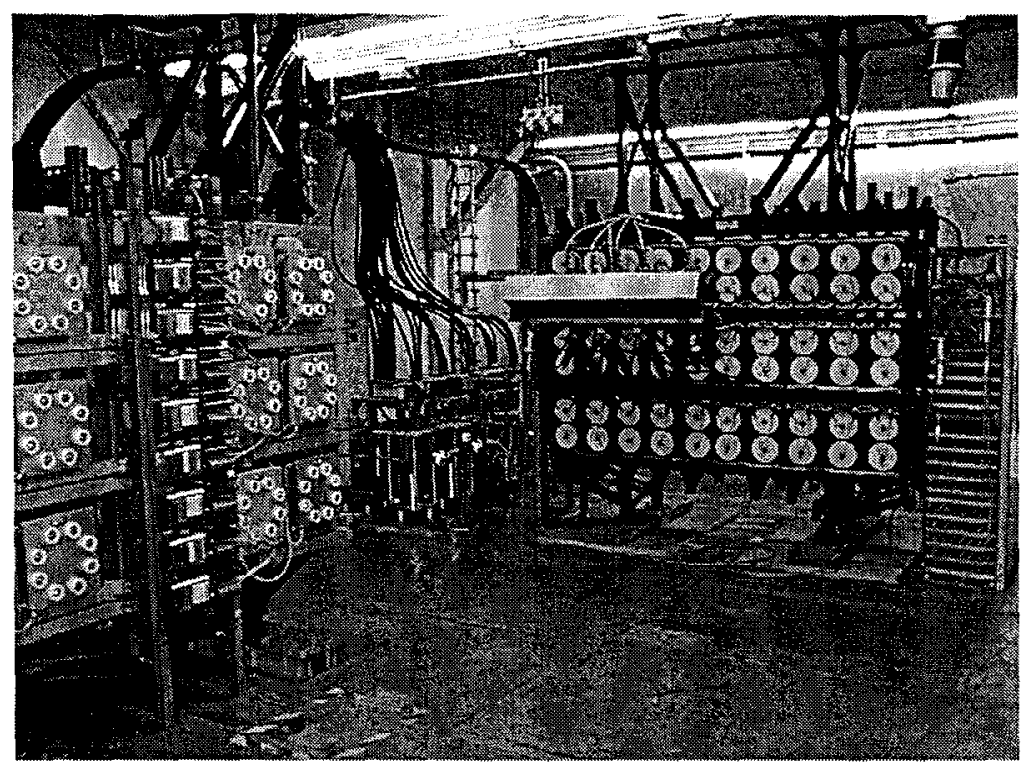

Fig. 5. The SSPX capacitor banks. 0.5MJ formation bank on left, 1.5MJ sustainment bank and pulse-forming inductor on the right.

Operation of the SSPX power systems and data acquisition is under computer control using a single screen graphical user interface (GUI). The power supplies used for SSPX had to be modified for remote control so that we can program the bank charging voltages, the ignitron firing times, and the magnet supply currents for the injector. This system has functioned reliably for over 2000 plasma discharges to date.

Incremental funded was awarded in January 1999 to design and install a set of six magnetic field bias coils for SSPX. The primary function of the bias coils is to provide flexibility in operation and the ability to optimize the magnetic configuration. They will allow operation with a lower threshold current for spheromak formation and sustainment, which should reduce the magnetic turbulence and energy transport. They will also allow operation with a divertor electrode or, with "reverse" current, provide a magnetic insulating layer between the spheromak plasma and the outer flux conserver surface in order to reduce impurity generation.

During the design phase, we used the CORSICA code to explore a number of possible coil configurations. The primary design drivers were the need to keep the flux surfaces parallel to the flux conserver in order to keep open field lines to a minimum, and the cost, which keep the number of coils to a minimum. In the end, we achieved these goals with a set of 6 coils put together in four assemblies. Two of the coil assemblies mount inside the vacuum vessel and two outside. The final, optimized magnet configuration and coil layout are shown in Fig. 6: 


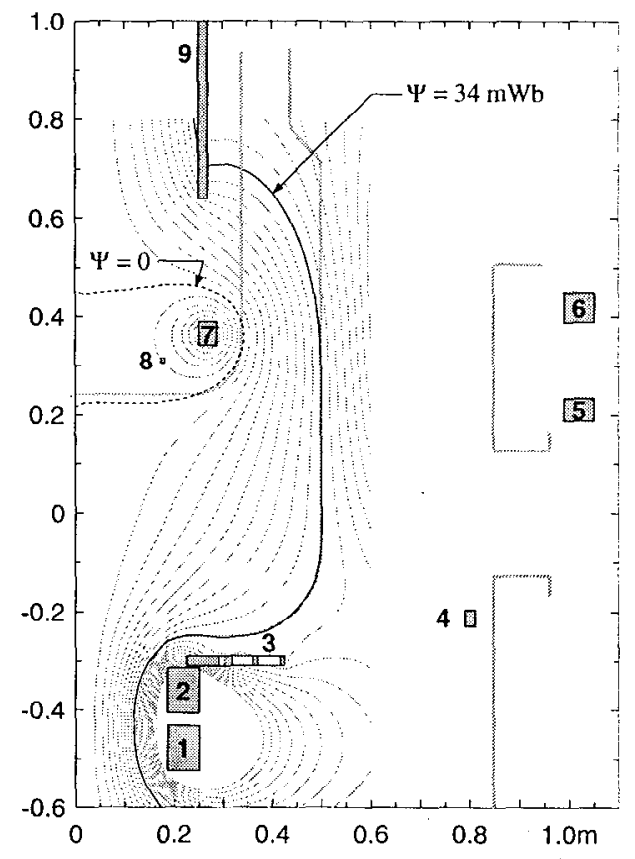

Figure 6 Vacuum magnetic field with the bias magnetic coils. The case shown carries the full gun flux from the injector to the divertor. The design has been optimized so that the field lines are tangent to the flux conserver, thus minimizing field errors and resulting loss of helicity.

At this time, we have fabricated the coils and they are ready for installation in the machine. We have also installed six high current power supplies that we obtained from the ATA facility. These supplies are ready to connect to the coils once the control circuits have been modified for computer control. Fig 7 shows both the coils and the power supplies.
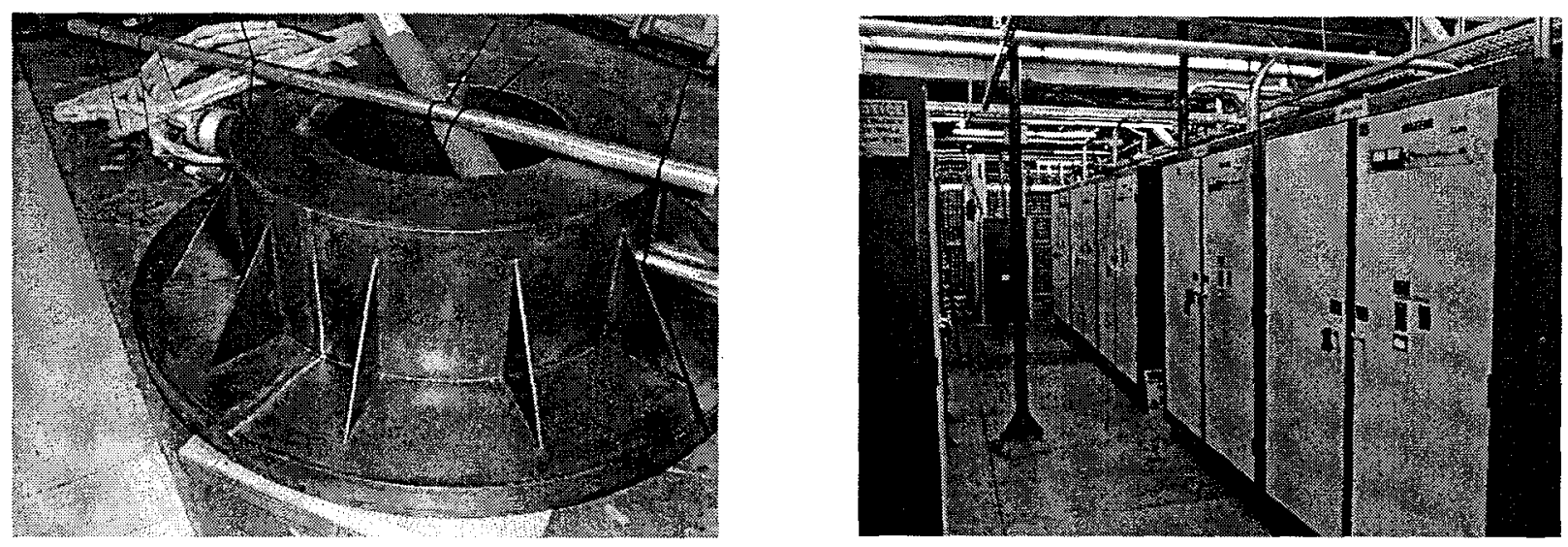

Fig. 7. Bias coils ready for installation (RHS) and bias coil power supplies (LHS). 


\subsection{Plasma Diagnostics}

A large suite of diagnostics is planned for SSPX to measure basic discharge and plasma properties, magnetic fields, and internal profiles required for transport studies. Before plasma experiments started, we designed and tested dual-axis magnetic probes, a gated plasma TV camera, designed and tested an Ultra-short-pulse reflectometer, and began design and fabrication of a Transient Internal Probe in collaboration with the University of Washington. A brief status report of each of these systems follows.

\section{Magnetic probes}

An array of fourteen magnetic field probes is mounted in the flux-conserving wall as a diagnostic to evaluate the field properties of the spheromak. These probes consist of orthogonal loops to measure both the toroidal and poloidal fields. The effect of currents in the wall is removed by a measured Fourier transform response function. The process is similar to that used successfully elsewhere. The probes are grouped as a toroidal array to measure asymmetries and a poloidal array to determine the basic spheromak fields and currents.

The CORSICA code has been used to determine the inversion of probe data to obtain SSPX parameters such as the total toroidal current (sum of that inside the separatrix and in the edge plasma), the toroidal current inside the separatrix, and the internal inductance. A large set of ideal, zero-beta MHD equilibria were calculated yielding sets of spheromak parameters and wall magnetic probe "data." Fit parameters were found for the SSPX parameters in terms of the magnetic probe signals, using 8 in the initial poloidal array of 14 . (The eventual array can have up to 2 poloidal arrays of 19 probes each, and an additional 8 probes in a toroidal array.) The edge value of $\lambda=\mu_{0} \mathbf{j} \bullet \mathbf{B} / B^{2}$, which can be calculated from the ratio of injector current and flux $\left(\lambda_{\text {edge }}=\mu_{0} I_{\text {gun }} / \Psi_{\text {gun }}\right)$, was also used as input.

The fitting used the singular-value decomposition method of a least-squares fit so as to allow elimination of near-singular independent data, that is, data which will contribute little precision to the inversion but which can make a large contribution to errors. Obviously, the validity of the model will need to be checked by careful analysis of the experimental data during experimental operation. The results of applying this 
process to discharge parameters is used in an IDL program to invert the experimental data.

\section{Ultra-short pulse reflectometer.}

Electromagnetic waves reflect from critical layers in the plasma where the plasma frequency equals the wave frequency and where the "right-hand cutoff" frequency (a combination of plasma and electron cyclotron frequencies) equal the wave frequency. The distances to these layers can be measured by injecting short pulses of the orthogonal polarized (ordinary " $\mathrm{O}$ " and extraordinary " $\mathrm{X}$ ") waves into the plasma and measuring the time delays for the reflections. This then yields the profiles of density and magnetic field. In addition, the rotation (shear) of the spheromak magnetic field causes a coupling between the two polarizations, so in principle the vector magnetic field can be determined.

We have been doing extensive modeling of the process using the CORSICA code, and in a collaboration with UC Davis are constructing an instrument to make the measurement. There will be many (up to 64) frequencies to map out the density and magnetic field profiles.

The modeling for the waves[2] uses a fully electromagnetic code (both 2 and 3 dimension versions) coupled to the CORSICA code which generated examples of the plasma equilibria. The results were compared to calculations using an analytic model based on the WKBJ wave approximation, and inversion techniques developed for use in the laboratory to generate a fast inversion of data. The inversion of experimental data can also be verified for particular cases using the code. The measurement technique may also be used to measure magnetic fluctuation amplitudes, important for the dynamo that drives the current in the plasma and determines energy confinement times.

Representative frequency channels of the actual instrument have been bench tested and the ordinary-mode system is almost complete. Shown in Fig. 8 is a measure of the reflection from an aluminum plate as a function of the distance 


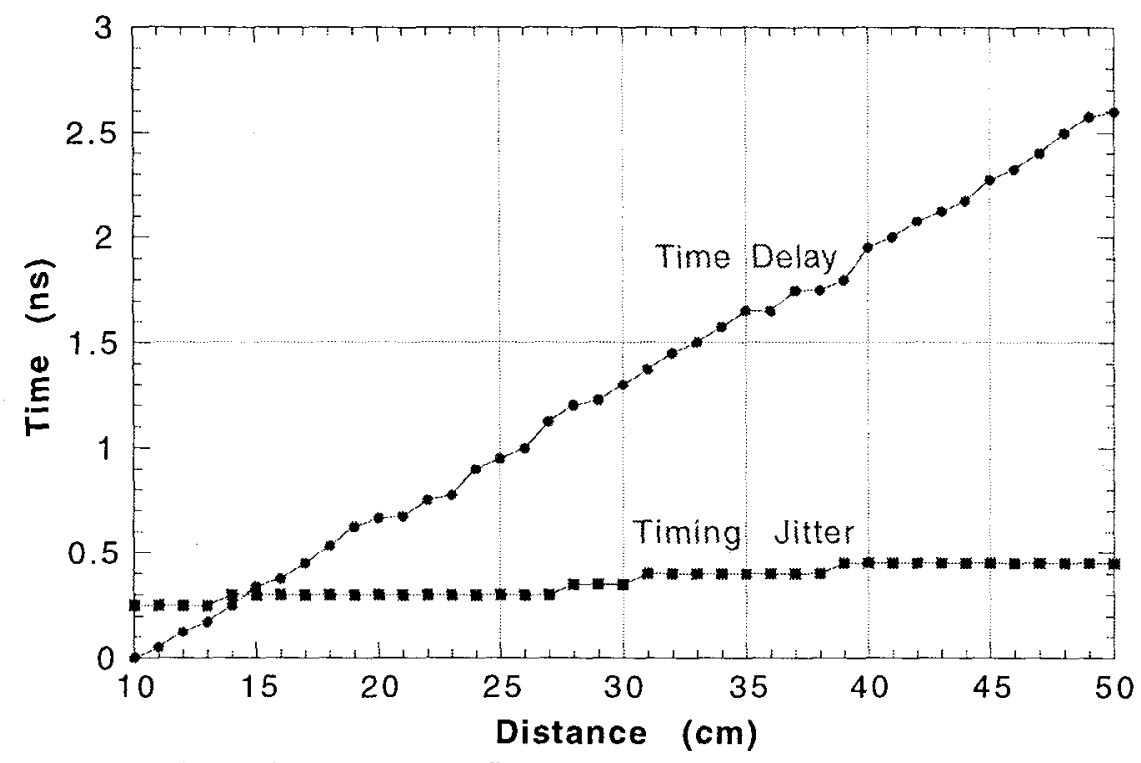

Fig. 8. Ultra-short pulse reflectometer test measurements showing delay time vs. distance from horn to reflecting plate.

\section{Transient Internal Probe (TIP).}

Accurate measurement of the internal magnetic field profile in SSPX is critical to understanding spheromak physics. The external magnetic probes do not provide a reliable measure of the internal field profile in a plasma with roughly circular cross section such as SSPX. Therefore, we started a collaboration with the University of Washington, Seattle, to design and install a TIP on SSPX. The Transient Internal Probe directly measures the internal toroidal magnetic field along a chord tangent to the magnetic axis[3]. The measurement is obtained by using a gas-gun to fire a glass bullet (constructed of material with a high Verdet constant) across the plasma in about $250 \mu \mathrm{sec}$. Measurement of the rotation of the plane of polarization of laser light reflected from the glass determines the local magnetic field. Although this diagnostic cannot be used every plasma discharge, the direct measurement will be used to check the accuracy of the other magnetic field diagnostics as well as directly providing data for physics analysis.

At this time, we have a graduate student from the UW on site working to install the probe. All the major components were fabricated at UW and the hardware was shipped to LLNL in September 1999. Subsequent to its delivery, we have been carrying out the necessary engineering reviews to document the safety of the device. Having done this, the next step is to test it in a side lab. We expect to be operating this instrument starting 
in the summer of 2000. Figure 9 shows a cross section of the TIP and SSPX; as can be seen, it is a rather large diagnostic, though the cost is quite modest.

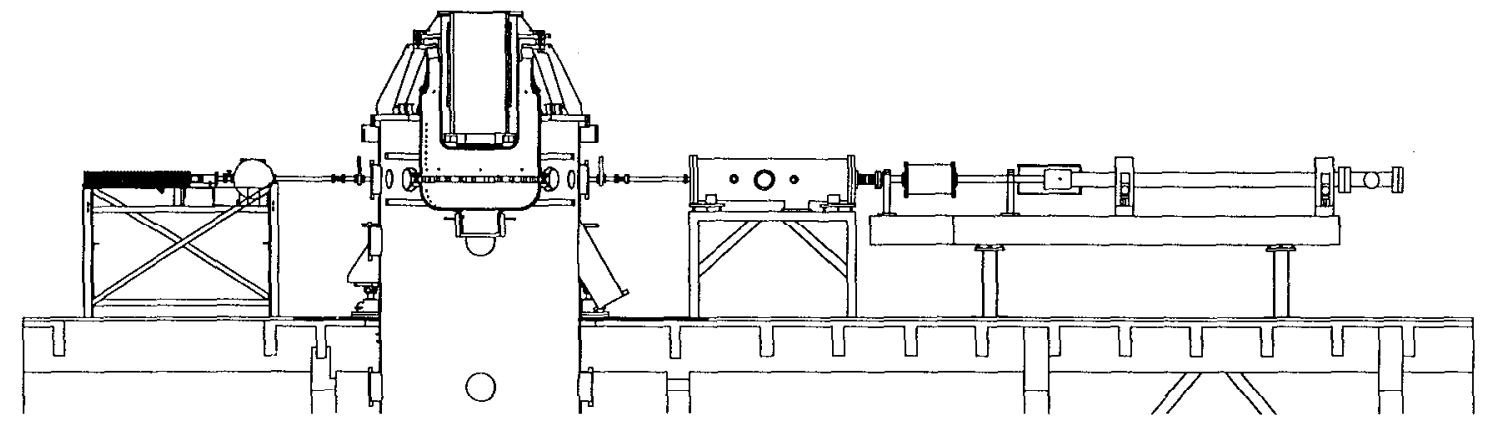

Fig. 9. Drawing showing Transient Internal Probe mounted on SSPX. Gas gun is on the RHS, catcher tank is on LHS.

\section{Data Acquisition}

The data acquisition system for SSPX now acquires some 100+ data channels containing about $4 \mathrm{MB}$ total data. We spent some effort to design a data acquisition system for SSPX, which will allow remote experimental site (RES) operation by scientists at other institutions. The LLNL MFE group has pioneered this concept as part of their collaboration on the DIII-D tokamak facility and we wanted to extend this capability to SSPX. In practice, this means we designed the instrumentation and controls to allow a scientist at a remote site to carry out experiments with the same effectiveness as if he were here at LLNL. This requires video and audio interaction, Internet physics data transfer, and shot sequencing and control data exchange.

\section{LLNL Spheromak Research Milestones}

A list of milestones for spheromak research at LLNL is included as Table 1 bclow. The status column shows the dates expected for the milestones compared to what we had orginally planned. Much of the delay in startup was due to difficulties with the outside vendor delivering the flux conserver and injector assembly. In the end, we provided technical direction to the vendor so that they could complete the system to specification. 
Table 1. Milestones for spheromak research at LLNL

\begin{tabular}{|c|c|c|c|}
\hline \multicolumn{2}{|r|}{ Milestone } & Date & Status \\
\hline 1. & $\begin{array}{l}\text { Race Facility Cleanup and Renovation } \\
\text { (OFES) }\end{array}$ & Complete & Complete \\
\hline 2. & $\begin{array}{l}\text { Complete detailed physics design of } \\
\text { flux conserver and helicity injector } \\
\text { (LDRD) }\end{array}$ & Complete & Complete \\
\hline 3. & $\begin{array}{l}\text { Install vacuum vessel and pumping } \\
\text { system (OFES) }\end{array}$ & October 1997 & Complete \\
\hline 4. & $\begin{array}{l}\text { Complete engineering design of flux } \\
\text { conserver and injector (LDRD) }\end{array}$ & January 1998 & Complete \\
\hline 5. & $\begin{array}{l}\text { Install Capacitor Banks and pulse } \\
\text { forming network (OFES) }\end{array}$ & August 1998 & Complete \\
\hline 6. & $\begin{array}{l}\text { Complete construction of flux } \\
\text { conserver and helicity injector (OFES) }\end{array}$ & Āugust 1998 & $\begin{array}{l}\text { Completed } \\
\text { March } 1999\end{array}$ \\
\hline 7. & Complete construction (OFES) & August 1998 & $\begin{array}{l}\text { Completed } \\
\text { November } 1998\end{array}$ \\
\hline 8. & $\begin{array}{l}\text { Install autonomous data acquisition } \\
\text { system (LDRD) }\end{array}$ & September 1998 & Deferred \\
\hline 9. & $\begin{array}{l}\text { Complete installation of basic set of } \\
\text { startup diagnostics (LDRD) }\end{array}$ & October 1998 & $\begin{array}{l}\text { Complete } \\
\text { April } 1999\end{array}$ \\
\hline 10. & $\begin{array}{l}\text { First discharge with machine } \\
\text { diagnostics (LDRD) }\end{array}$ & October 1998 & April 1999 \\
\hline 11. & $\begin{array}{l}\text { Start Phase II sustainment physics } \\
\text { diagnostics (LDRD) }\end{array}$ & November 1998 & April 1999 \\
\hline 12. & Design Bias Coils & September 1999 & April 1999 \\
\hline 13. & Fabricate Bias coils & October 1999 & October 1999 \\
\hline 14. & Install Bias coils & February $200 \overline{0}$ & September 2000 \\
\hline 15. & Demonstrate flux core configuration & December 2000 & \\
\hline 16. & Report on Configuration studies & September 2001 & \\
\hline
\end{tabular}

\section{Experimental Results}

The SSPX spheromak began operating April 2, 1999 after a four-month period of pre-ops testing and final hardware/diagnostics installation. Since then, more than 2000 shots have been obtained. We average about 25-30 plasma pulses per run day. Since we began routine operation, we have carried out experiments to characterize spheromak formation in SSPX, and are now working to increase the fraction of input energy going into building the stored energy in the spheromak plasma. At present, the plasma electron temperature in the spheromak is low, about $20-40 \mathrm{eV}$ instead of the desired $100+\mathrm{eV}$. Our data suggest this is the result of high-density operation coupled 
with impurity contamination likely produced during the initial gas breakdown. Therefore, work is underway to add titanium gettering of the flux conserver and to improve the glow discharge cleaning system.

We still have to bring the sustainment bank on line, which will increase the energy storage by a factor of three. Operation of this system has been delayed to component failures. Based on present performance, we expect that the pulse length and peak internal stored energy should at least double. We are also modifying the gas fueling system to increase the hydrogen density in the injector before breakdown, which should reduce impurity generation. The recent commissioning of the $\mathrm{CO} 2$ interferometer and SPRED VUV survey spectrometer have helped us to understand how the device is operating and how we can modify it to improve its performance.

During the FY99 operations period, we carried out experiments aimed at quantifying the formation threshold current and comparing with theory, measuring the edge fields and relating these data to the classical spheromak model for current and field profiles, and examining field buildup using both energy and helicity balance. These results are summarized below.

\section{Threshold studies}

Theory predicts that there is threshold current for ejecting a spheromak plamsa from the injector region. Basically, the radial current density must be sufficiently high that $j \times B_{\text {tor }}$ forces are large enough to pull the radial vacuum field lines (see 2 above) out of the injector region.

The data in Fig. 10 below show that the critical ejection current density $\lambda=j / B$, is set by the gun eigenvalue - determined by the geometry of the gun $\sim 20 \mathrm{~m}^{-1}$. This critical ejection $\lambda$ is determined at the moment that the gun-flux starts to be pulled out of the gun, characterized by a sharp rise in gun voltage. This calculation was further complicated by the fact that only a fraction of the total gun-flux was ever drawn from the gun during formation. This injected flux can be calculated from a field coil mounted at the mouth of the gun assuming a linear field profile, hence we find, 


$$
\lambda_{c r i t}=\frac{\mu_{0} I_{i n j}}{\psi_{i n j}}=\mu_{0} \frac{I_{\text {thresh }}}{2 \pi \int_{0.42}^{0.5} r B_{p}(r) d r}
$$

where $\mathrm{I}_{\mathrm{inj}}$ is the injector current, ping the injector magnetic flux, and $\mathrm{I}_{\mathrm{thresh}}$ the threshold injector current for ejection. $B_{r}$, the radial field in the electrode gap, is obtained via a linear extrapolation across the electrode gap spanning $0.42 \mathrm{~m}$ to $0.5 \mathrm{~m}$.

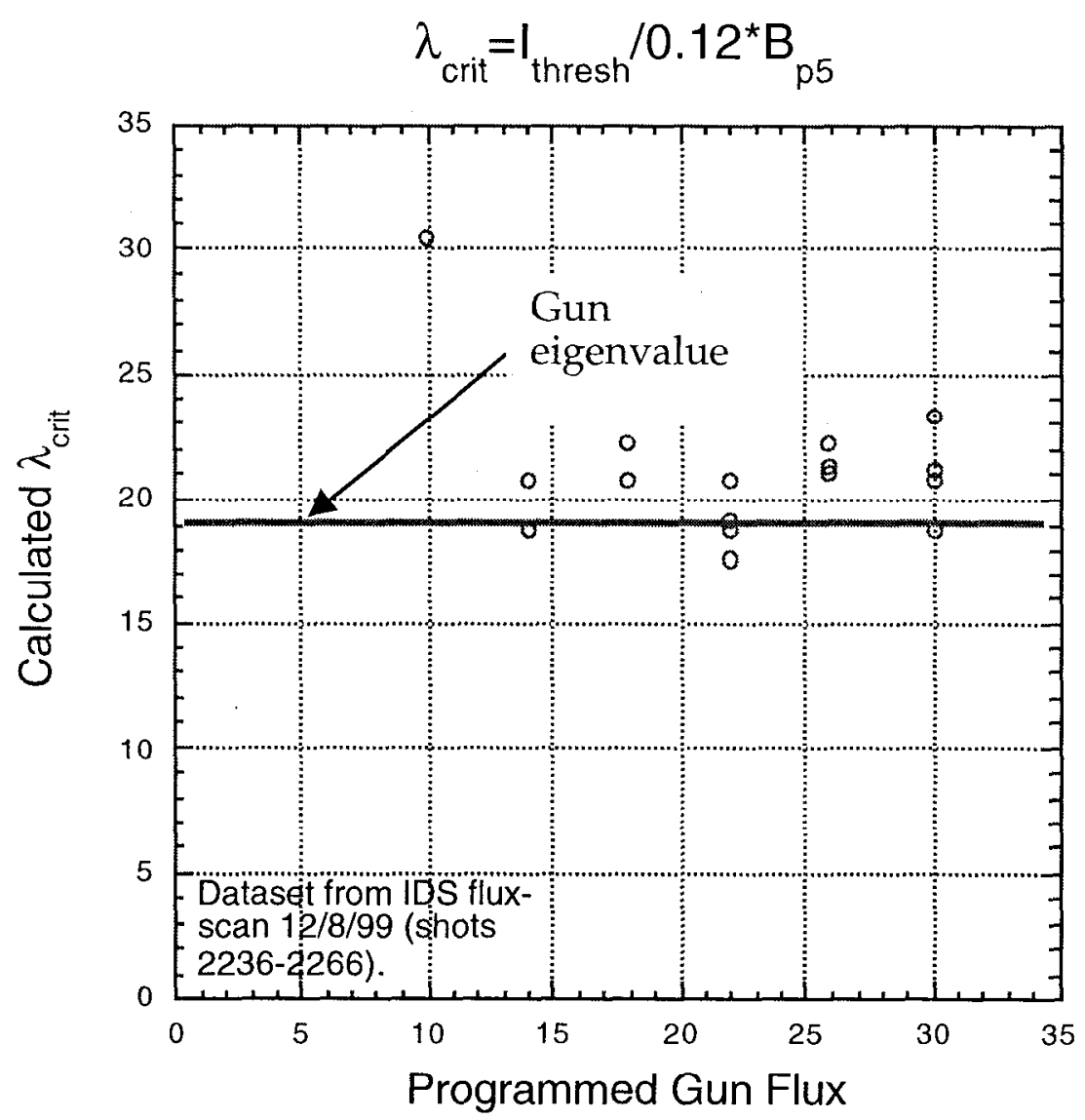

Figure 10. Experimental injection thresholds agree with theory.

The 'bubble-burst' condition just described is observed only for low $\lambda$-gun shots at high gun- $\lambda$ (corresponding to below $14 \mathrm{mWb}$ for these shots), large fluctuations in both gun-voltage and gun-flux suggest that the ejection occurs as a series of discrete events. This multiple-ejection scenario is presently being explored in the context of a current-drive conjecture proposed by M. Nagata of the Himeji Institute of Technology 
in Japan, in which multiple spheromaks eject from the gun and merge with the main spheromak to build helicity there.

\section{Benchmarking CORSICA to measured spheromak edge fields}

The CORSICA code (a $2 \mathrm{D}$ resistive MHD code) has been used to determine the equilibrium at various instances during the discharge by fitting to edge fields - both during build-up and in decay. Figure 11 shows how the measured poloidal magnetic field around the boundary compared to that calculated by the CORSICA code. The circles in the drawing on the left indicate the location of the probes around the flux conserver. The box on the right shows the measured fields (dots and + ) compared to CORSICA (solid line). The solid circles indicate the location of selected probes.
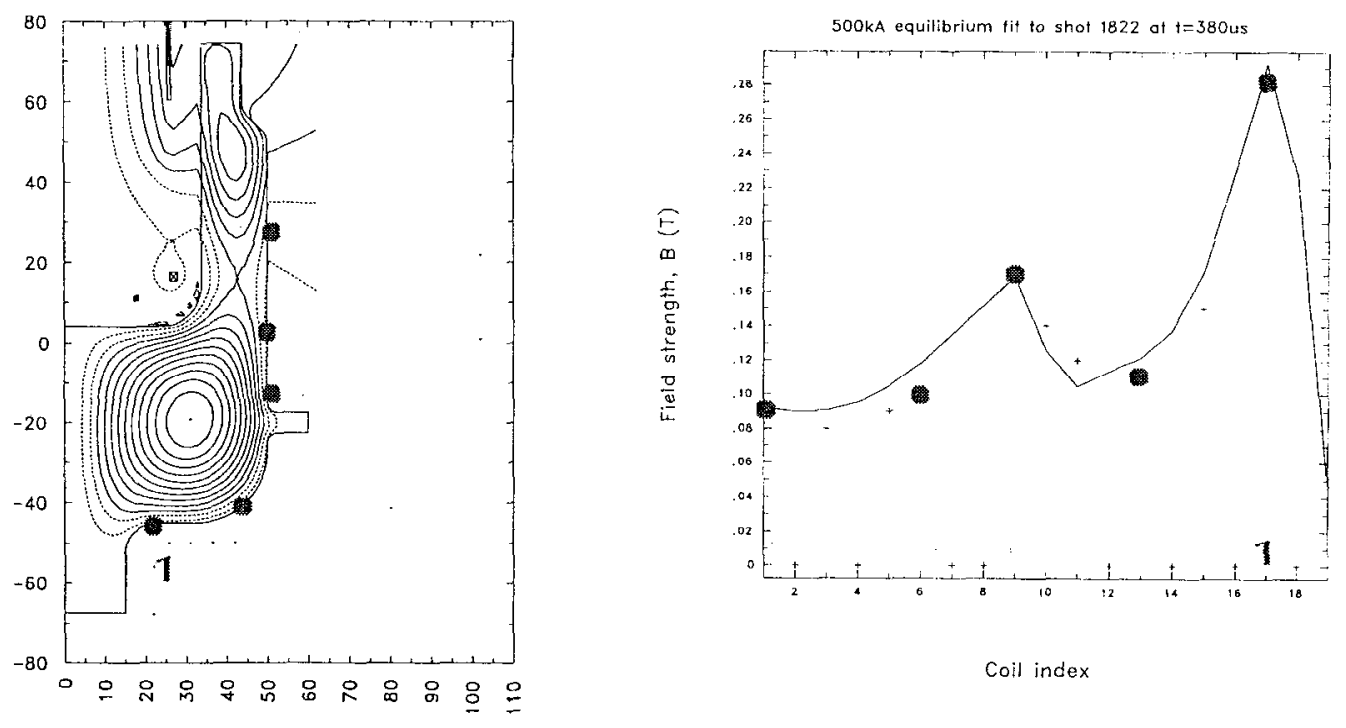

Fig. 11. Calculated MHD equilibrium from the CORSICA code. LHS-SSPX cross section with computed field profiles showing location of probes. RHS-comparision of measured and calculated (line) edge poloidal fields.

In general, the agreement is quite good. In this particular case, the current density on the open field lines is twice that on the closed field lines, indicative of the buildup period during which helicity is transported from the edge plasma to the core.

During the decay phase of a spheromak pulse, when the plasma in the flux conserver is disconnected from the injector, we expect that the plasma currents and fields will more closely resemble a Taylor relaxed (minimum energy) state. As time 
progresses and the edge plasma cools further, the plasma will deviate from this ideal state.

Our data do indeed show that at the beginning of decay - at the time that the spheromak has reached a Taylor state - the internal field profiles are identical to those predicted by the Bessel function solution to $\nabla \times B=\lambda B$. That is, CORSICA has been benchmarked against the BFM (see Fig. 12 below). During the peak of sustainment, CORSICA suggests a total toroidal current of $550 \mathrm{kA}$ - for shots at $7 \mathrm{kV}, 22 \mathrm{mWb}$ programmed flux (standard operating conditions). Fitting routines have recently been developed, such that the total internal plasma current is varied until an error function is minimized. A routine is being developed to fit internal lambda profiles
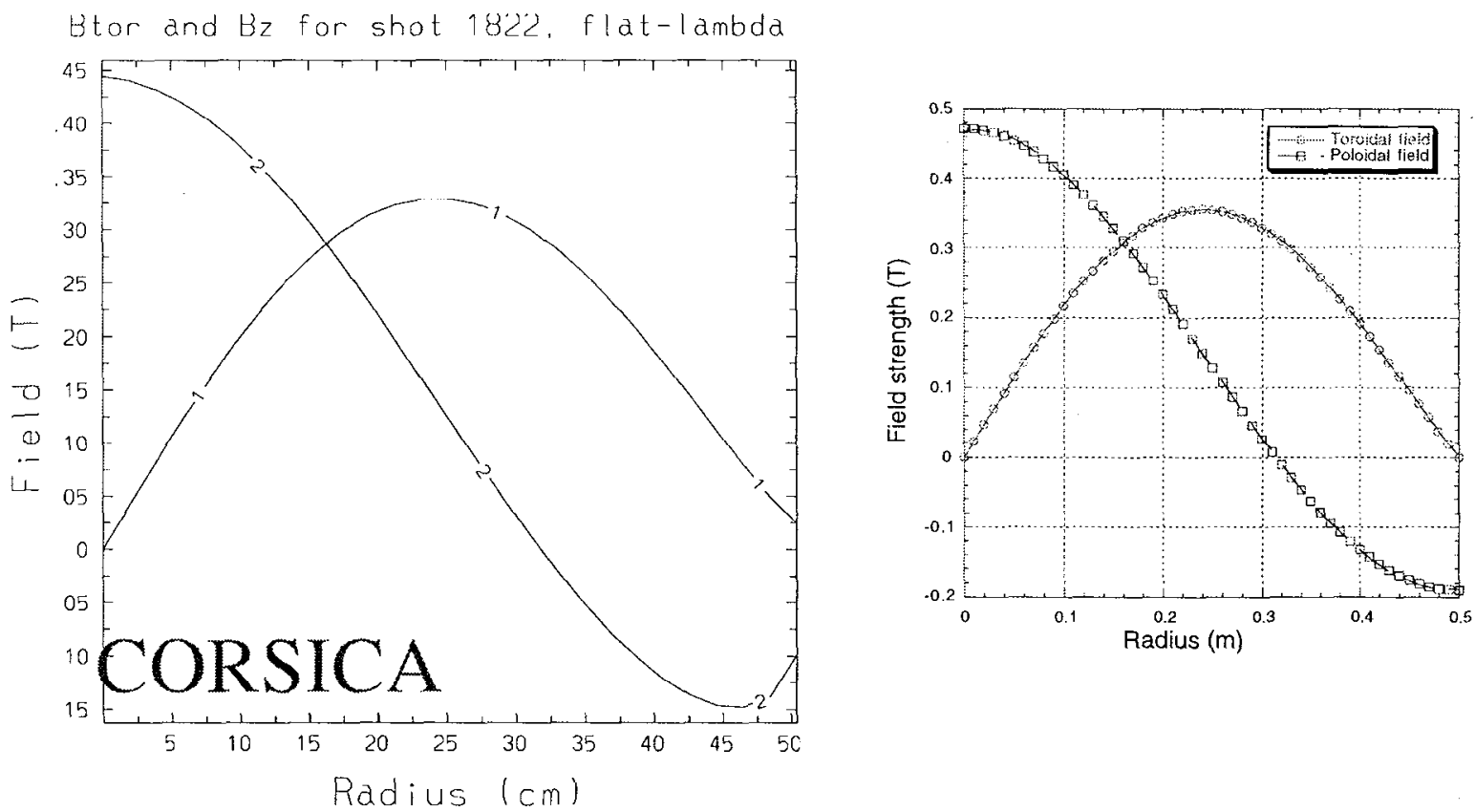

Fig. 12. (a-on left) Internal field profile for SSPX plasma inferred from magnetics data using the CORSICA code. (b-on right) Predicted profiles for a Taylor relaxed state using the Bessel Function solution for SSPX geometry. 


\section{Helicity Build-up calculations}

The helicity of the spheromak can be calculated by two means: firstly, from the solution to $1^{\text {st }}$ order differential form of the helicity balance; and secondly, by using CORSICA to compute the helicity of a given equilibrium and hence provide a calibration constant to convert the measurement of an edge field to 'helicity'. The two methods agree remarkably well, hence it is safe to use the differential helicity balance as a model to predict the evolution of future experiments. Shown below in Fig. 13 is the predicted helicity evolution for sustained operations. Implicit is the assumption that burn-through has occurred early in the discharge, hence a conservative helicity decay time of $600 \mu \mathrm{s}$ is used (observed in SPHEX and CTX discharges). This calculation is sensitive to the amount of flux drawn from the gun; hence it can be expected that total plasma currents in excess of 1MA will be achieved with bias coils installed.

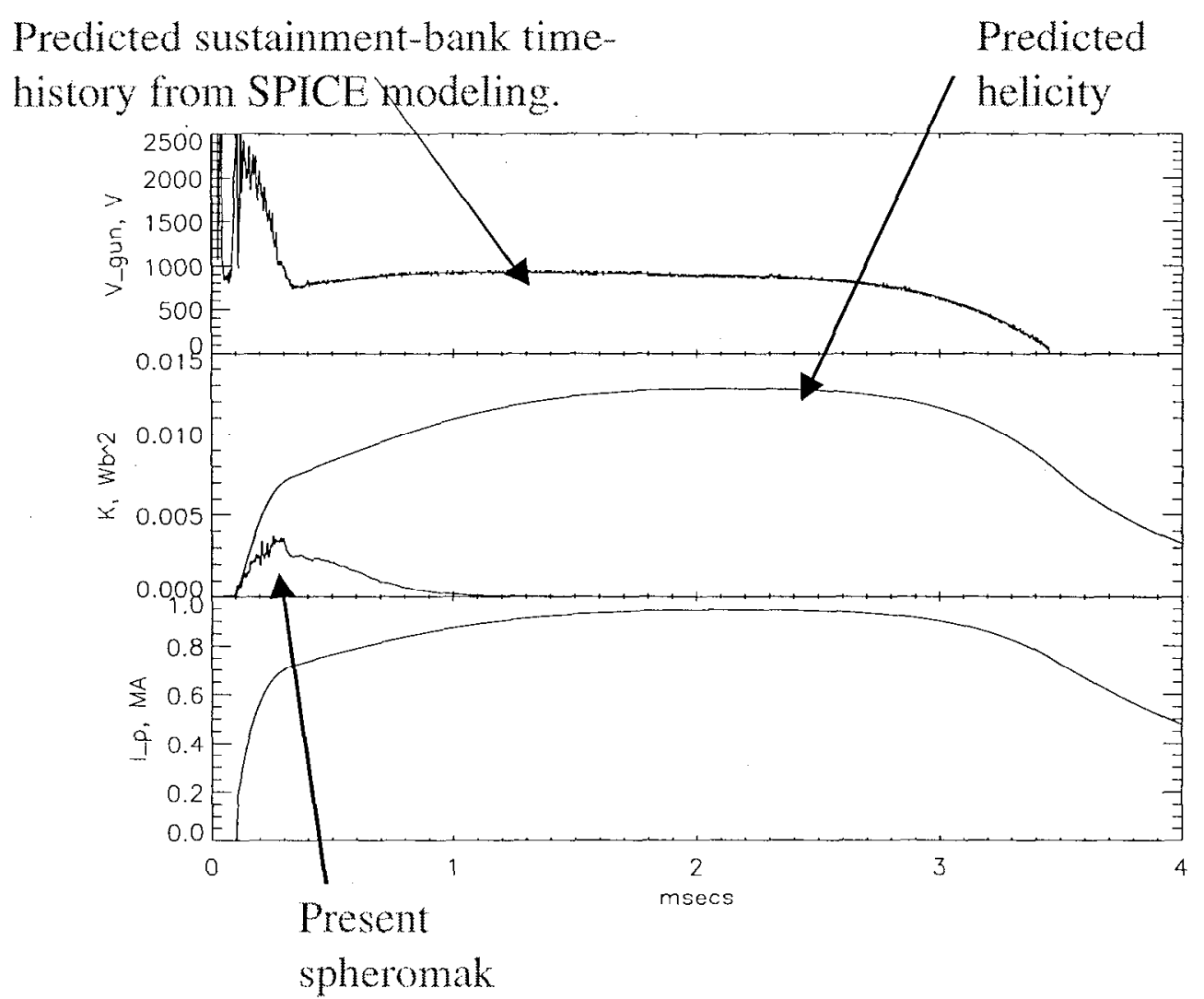

Fig. 13. Helicity is used to predict sustained spheromak evolution. 


\section{Flux-core spheromak simulation experiments}

A remarkable facet of the gun-injected spheromak is that the efficiency is set largely by the ratio of spheromak- to gun-lambda. Ultimately with the bias coils in place it will be possible to draw all of the programmed gun-flux into the flux-conserver and in the process force the gun-lambda to be equal to the spheromak-lambda. This condition will give rise to the most efficient operations. In the absence of the bias coils, some experiments have been performed to lower lambda-gun - simply by reversing the current in a coil that would otherwise buck the solenoid flux radially across the gun, it is possible to draw this flux into the FC, hence lower lambda gun.

Fig. 14 shows the plasmas created in these experiments. On the left is the standard case, which can be identified by the fact that all of the field lines entering the flux conserver in the gun also exit there. On the right, the modified case has vacuum field lines passing from the inner electrode down and out through the flux conserver. The difference in the resulting plasma cross section is clearly evident.
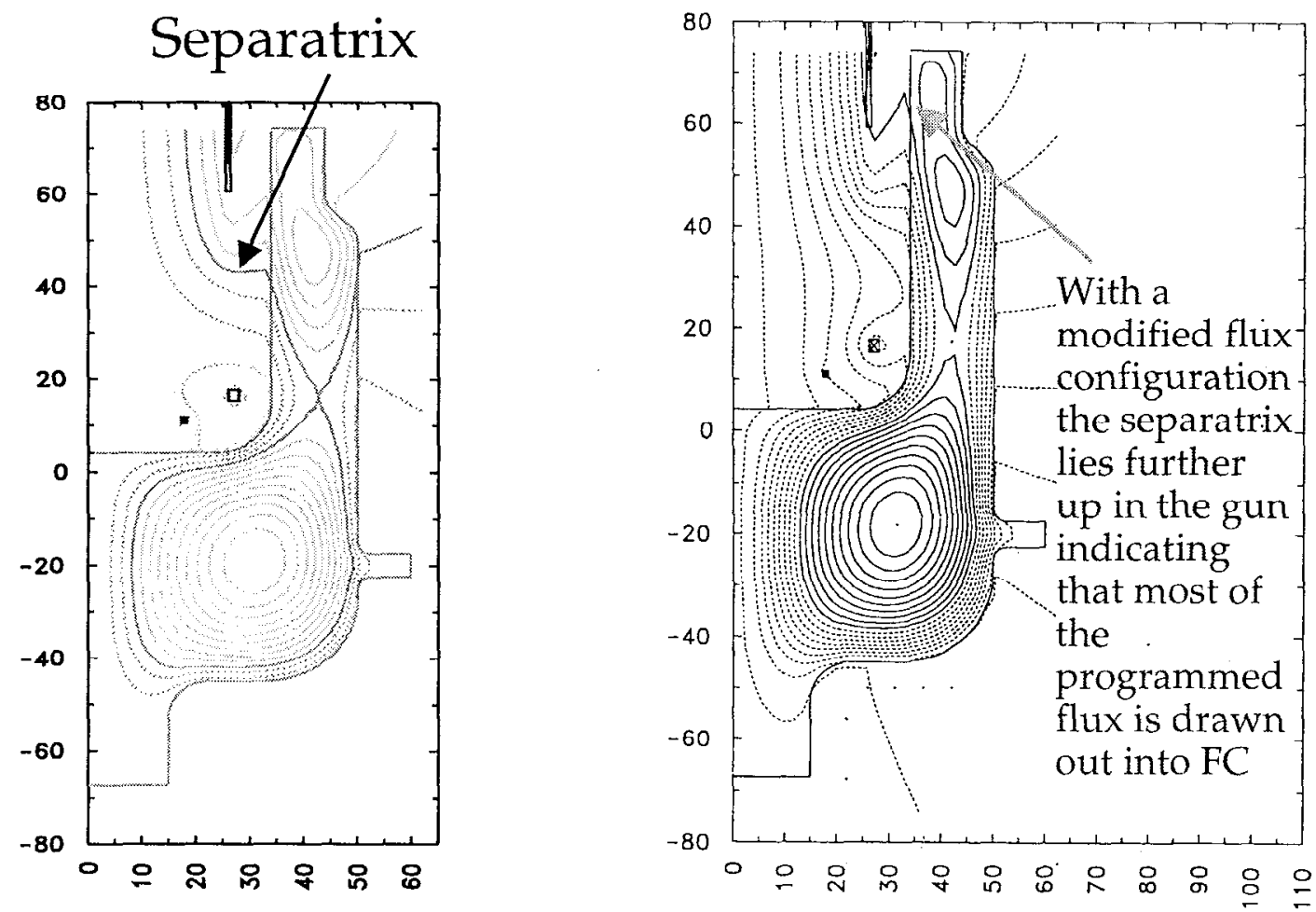

Fig. 14. Modified flux core experiments. LHS-standard configuration. RHS-modified flux-core configuration. 
Initial experiments performed in this configuration point to increased efficiency (field-energy/gun-input-energy), longer decay times, and lower fluctuation levels. Plotted below in Fig. 15 is an efficiency curve for modified flux operations compared with standard flux - it is clear that by closer lambda matching, the device efficiency can be improved markedly.

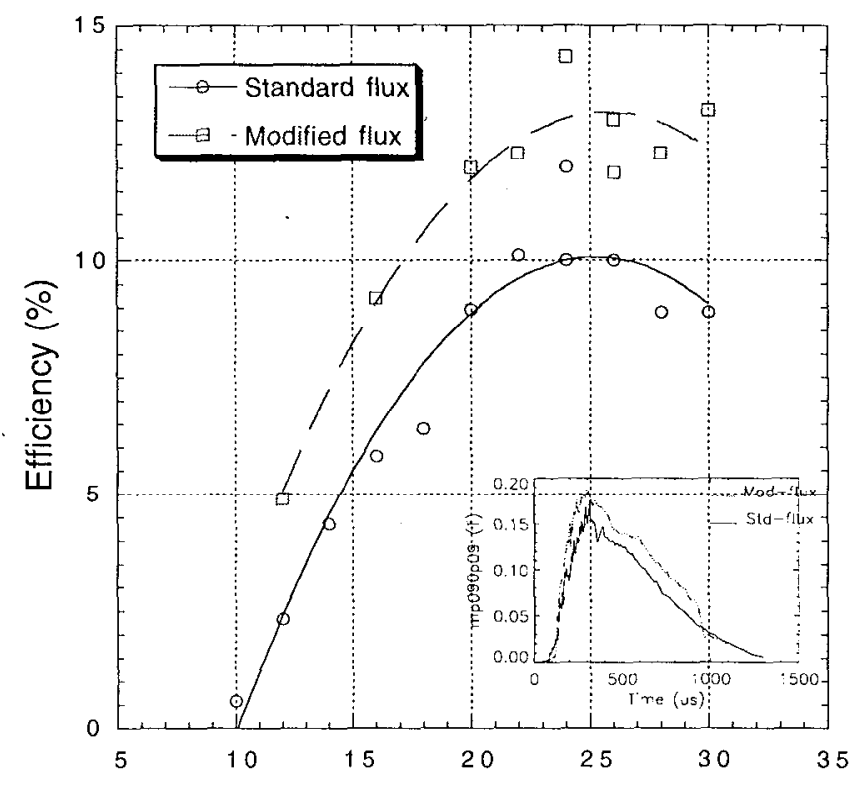

Fig. 15. Peak efficiency against programmed flux for two different initial flux configurations - with a partial fluxcore spheromak higher efficiency is achieved.

The efficiency is being investigated more intensively in terms of the modes or instabilities that are driven in each configuration through CORSICA modeling and mode analysis techniques. Furthermore the structure of the underlying turbulence is being explored for each operational mode.

\section{Preliminary sustainment studies with split formation bank}

By splitting the formation bank into two halves and firing each half independently it was possible to perform a limited investigation into sustained operations. The investigation was aimed at resolving a couple of issues for sustained ops: firstly - is it possible to sustain the spheromak at gun-lambdas lower than the critical ejection lambda (as previously thought), and secondly is it possible to continuously build-up field energy with a longer pulse. In answer to the first, it was found that the ejection threshold would need to be met to sustain the spheromak - this lead to a rethinking of the design of the sustainment bank. In answer to the second, it was appeared that a 
second pulse would simply replace the first if the delay between them exceeded a few hundred microseconds, however, the field energy would build if the delay were shortened. Data from such a double-pulse experiment is shown in Fig. 16 below.

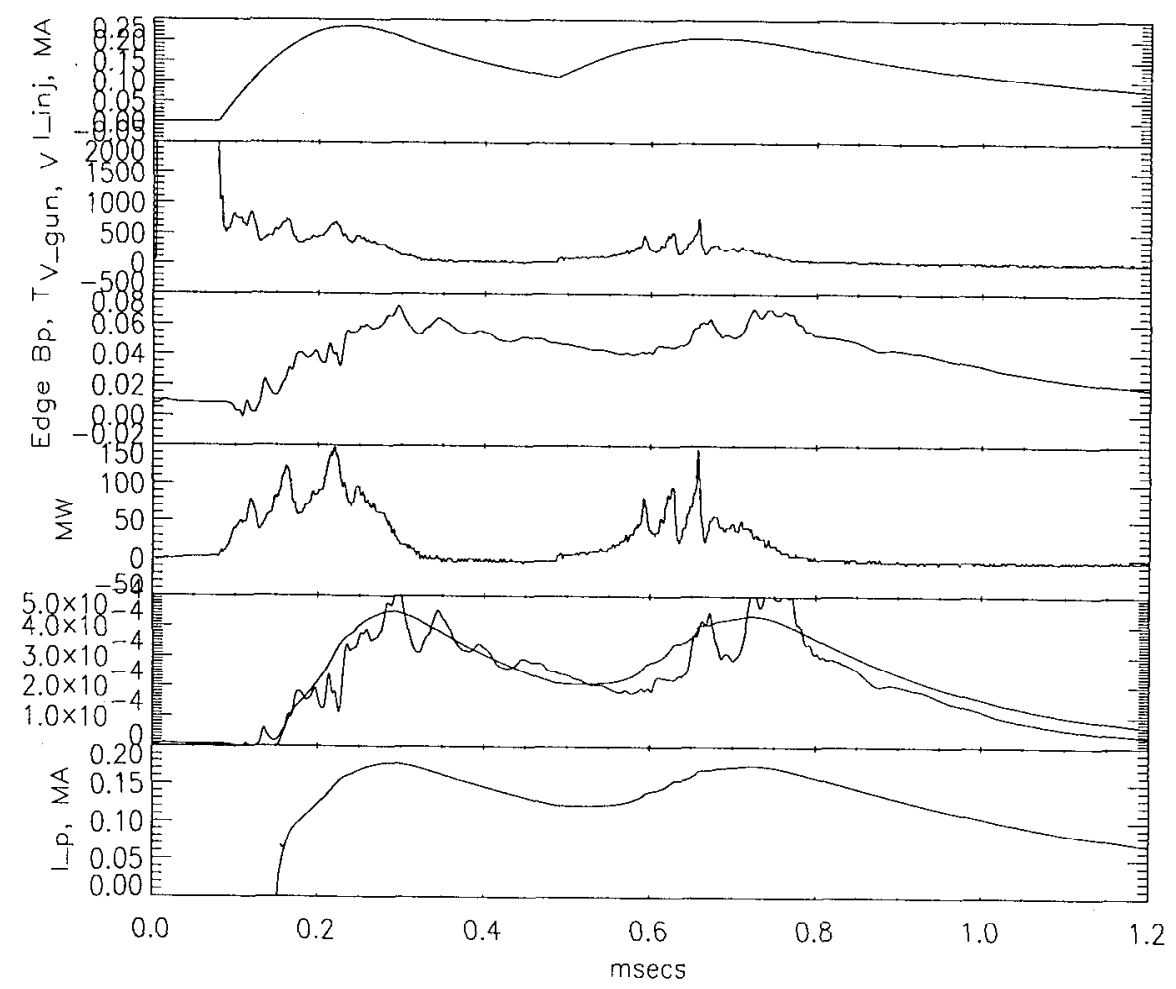

Fig. 16. Results of splitting the formation bank into two parts to simulation spheromak sustainment. A second spheromak injection occurs at $0.65 \mathrm{msec}$.

\section{Present Status and Future Directions}

At present, the SSPX spheromak device is operating routinely and we are carrying out formation experiments while bringing on line the remaining profile diagnostics needed to carry out energy confinement studies. We have over 2000 pulses so far. Peak plasma currents are in the range of $0.5 \mathrm{MA}$ with a $1 \mathrm{msec}$ duration. The magnetic field

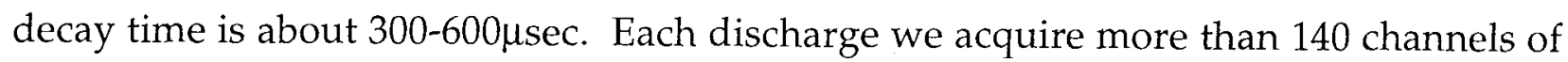
diagnostic data totally more than 3MB. The USDOE Office of Fusion Energy funds these activities. LDRD funds are supporting a new project to examine more fully the 
configuration space made possible by the installation of the bias field coils. These coils will be installed in FY2000.

The main focus of the plasma experiments now is to obtain clean spheromak plasmas with electron temperatures at or above $100 \mathrm{eV}$. Spectroscopic data indicates the presence of carbon, nitrogen, and oxygen impurities at higher than desired levels. In addition, density measurements show that we need to lower the plasma density about a factor of four. We are beginning a campaign utilizing titanium gettering to reduce recycling and better pump impurity gases. Based on results of breakdown experiments, we are modifying our gas injectors to increase the gas density in the injector. This should reduce the breakdown voltage and gas inventory in the chamber so that a cleaner, lower density plasma should be produced. In addition, we are bringing the sustainment bank on-line to increase the pulse length and plasma energy. Based on present results, we expect that the stored energy in the plasma could increase a factor of two or more with the longer pulses.

The data analysis used to interpret experimental results continues to mature. We routinely use the CORSICA code to determine the plasma parameters from the measured edge magnetic fields. We carry out Fourier transforms of the magnetic probe data to determine the amplitude and frequency of MHD modes so that we can correlate them with changes in the helicity injection or decay rate. We have applied a circuit analysis model to the spheromak power systems so that we can identify the energy losses in the system and improve the overall efficiency by adjusting the circuit impedance. We have compared the spheromak evolution from the point of view of both energy balance and helicity balance, and find pretty good agreement between these different approaches. We are starting to examine the density behavior to determine what is controlling the particle inventory and to learn how to reduce the density.

At this point, we had expected to be operating sustained plasmas above $100 \mathrm{eV}$. However, problems with the sustainment bank hardware (the high current electrical joints) have prevented us from using this system. In addition, we still working to understand how the plasma interacts with the tungsten wall. SSPX is the first spheromak to operate with tungsten-coated walls and the effect of the surface chemistry 
has not been documented. Consequently, we have had to invest more experimental time in particle-control experiments than originally planned.

During the next year we will be finishing key profile diagnostic systems, carrying out experiments with the new bias field coils, and measuring the confinement in sustained spheromak plasmas. The main new diagnostic to come on line in FY2000 will be the Profile Thomson Scattering system. With this diagnostic, we will be able to measure directly the electron density and temperature profiles at ten locations across the plasma at a single time point. These data will be essential in determining the energy confinement. The bias coils promise to open up exciting new operating modes for the spheromak; preliminary data partially simulating these configurations already show encouraging results.

During the course of the next eighteen months, we expect that the US DOE Office of Fusion Energy Science will support all spheromak research at LLNL. We have made significant progress in getting the experiment to the point where we can address the key issues with confidence. We note that SSPX is the first of the Alternate Concept facilities funded by the OFES in the FY1997 cycle to become operational and to produce scientific results.

\section{References}

1. T. K. Fowler, Fusion Tech. 29, 206 (1996).

2. B. I. Cohen, L. L. LoDestro, E. B. Hooper et al., Plasma Phys. Cont. Fusion 40, 75 (1998).

3. J. P. Galambos, M. A. Bohnet, T. R. Jarboe et al., Rev. Sci. Instrum. 68, 385 (1997). 


\section{Appendix 1. Recent Spheromak Publications}

B.I.Cohen, L.I.. LoDestro, E. B. Hooper and T.A. Casper, "Simulations of broadband short-pulse reflectrometry for diagnosing plasma density and magnetic field profiles," Plasma Phys. Control. Fusion 40, 75 (1998),.

Cohen, Bl; Hooper, EB; Kaiser, TB; Williams, EA; et al., "Modeling of ultra-short-pulse reflectometry," Physics of Plasmas 6, 1732 (1999).

Cohen, BI; Hooper, EB; Spang, MC; Domier, CW, "Theoretical aspects of the use of pulsed reflectometry in a spheromak plasma," Rev. Sci. Instrum., 70, 1407 (1999).

T. K. Fowler, D. D. Hua, E. B. Hooper R. W. Moir, L. D. Pearlstein, "Pulsed Spheromak Fusion Reactors," Comments Plasma Phys. Control. Fusion (Comments Modern Phys. Part C) 1(3), 83 (1999).

E. B. Hooper, L. D. Pearlstein, R. H. Bulmer, "MHD equilibria in a spheromak sustained by coaxial helicity injection," 39, 863 (1999).

Hooper, EB; Cohen, RH; Ryutov, DD, "Theory of edge plasma in a spheromak," J. Nuclear Mat. 278, 104 (2000).

K. I. Thomassen, E. B. Hooper, and D. D. Ryutov, "The Spheromak Path to Fusion," Workshop on Cost-Effective Steps to Fusion Power, Los Angeles, Jan 25-27, 1999; UCRL-JC-132914. 


\section{Appendix 2. Collaborations}

There are several experiments currently underway in the US which provide information on resistive MHD in related configurations. We are working with these groups to ensure that we obtain the broadest possible understanding of the data.

There are three experiments which are explicitly studying magnetic reconnection: Prof. M. Brown at Swarthmore is forming pairs of small spheromaks and examining the reconnection layer between them. Dr. M. Yamada at Princeton Plasma Physics Laboratory has been studying colliding spheromaks and using the measurements to examine the behavior of reconnection. Prof. P. Bellan at Cal Tech is generating high current arcs which mimic solar flares, including effects of reconnection and magnetic helicity.

Small spheromaks are formed and accelerated to high speeds (many $\mathrm{km} / \mathrm{s}$ ) for injection into tokamak plasmas for fueling. The primary US effort in this area is by $\mathrm{U}$. C. Davis and located in the same LLNL building as SSPX. There are related efforts in Japan.

Reversed field pinches are a magnetic field confinement geometry which is limited by resistive MHD modes similar to those in the spheromak, although with a different magnetic geometry. The primary effort on RFP's in the US is the MST at the University of Wisconsin at Madison, and a larger device is operating in Padua, Italy. We have a collaboration with the MST group to use their spectrometer on SSPX to examine plasma impurity content, as well as apply their turbulent transport diagnostics on SSPX at the appropriate time, in order to provide easily compared data on transport in the presence of magnetic turbulence.

In addition to these activities, we are collaborating with General Atomics. Their support was critical during FY1997-98 for the analysis of the stability of the SSPX geometry to ideal MHD modes. During the past year and in FY1999 they are providing support for the design of diagnostics, especially the Thomson scattering.

The University of Washington is building a Transient Magnetic Probe to measure the magnetic field in the spheromak. They have a graduate student on site full time and Prof. T. Jarboe provides significant physics support as well. 
Sandia National Laboratory is collaborating with SSPX to apply their extensive surface physics expertise to the experiment. They have provided glow discharge cleaning hardware and manpower for data analysis. $\mathrm{T}$

We also have a collaboration with Prof. M. Nagata of the Hemeji Institute of Technology in Japan. Prof. Nagata loaned us a high resolution spectrometer and visited here for two months while measuring the plasma rotation and ion temperature in SSPX. 